1 International Health Policy Programme, Ministry of Public Health, Nonthaburi, Thailand

2 Global Virome Project, University Research Co., USA

Cite this as: BMJ2022;376:0311 http://dx.doi.org/10.1136/bmj.o311

Published: 04 February 2022

\section{Resilient and equitable recovery from the covid-19 pandemic}

\author{
Viroj Tangcharoensathien, ' Dennis Carroll, ${ }^{2}$ Angkana Lekagul'
}

The emergence and the impact of the covid-19 pandemic have been driven by the interactions of multiple determinants such as ecology, socio-economic status, inequality, and politics leading many commentators to write about how we should consider covid-19 to be a syndemic rather than a pandemic.

Covid-19 has uncovered deeply rooted social inequities and brought racial injustice to the forefront of public health and political attention. Several studies have shown that ethnic minority populations had a greater risk of covid-19 infection and mortality. ${ }^{1}$ This disproportionate impact of covid-19 on ethnic minorities is partly explained by socio-economic factors, ${ }^{2}$ however, as Razai et al have written, cultural and structural racism are also causes. ${ }^{3}$ In the US, underpinned by long-standing structural and societal inequalities, living and working conditions predispose US minority communities to worse outcomes from pandemic. ${ }^{4}$

This pandemic is further complicated by man made disasters. Politicising and ignoring science has hampered the rollout of timely and effective control measures ${ }^{5}$ while political conservatism in the US inversely associates with perceived health risk and adoption of health protective behaviours. ${ }^{6}$ Furthermore, hate speech has triggered anti-Asian racism and xenophobia in the $\mathrm{US}^{7}$ resulting in an increased level of post-traumatic stress disorder among Asian and Asian American young adults, who reported verbal or physical assault. ${ }^{8}$

Around one million excess deaths occurred in 2020 in 29 high income countries, and men have higher age standardised excess death rates than women in almost all countries. ${ }^{9}$ A study on excess mortality spanning 79 high, middle- and low-income countries shows that the privatisation of healthcare, an underfunded health sector, and slow covid-19 containment and mitigation actions are key drivers of excess deaths. ${ }^{10}$ Timely activation of comprehensive responses, adapting health systems' capacity, preserving health systems' resources, and reducing health systems' vulnerability all contribute to effective country responses. ${ }^{11}$

However, untimely and ineffective containment measures against covid-19 have resulted in surges of cases and have overwhelmed healthcare systems around the world. This has knock-on impacts on access to non-covid essential health services, such as services for acute myocardial infarction, stroke, accident and trauma. A study in the US shows substantial and higher concentration of excess deaths from non-covid-19 causes among men and ethnic minorities, especially Black and non-Hispanic males, than covid-19 deaths. ${ }^{12}$ Lack of insurance coverage and overwhelmed health systems are causes of excess deaths from covid-19 and non-covid-19 conditions further widening the socio-economic divide of the pandemic.

This collective evidence from the pandemic should guide us on why we need to achieve a resilient and equitable recovery and how we might do this. We suggest the following:

Firstly, we must roll out covid-19 vaccination to at least $70 \%$ of world's population by 2022 . This is currently hampered by the global inequity in access to a covid-19 vaccine and the emergence of variants of concerns. As of 22 January 2022, only $14.4 \%$ of the population in Africa had received at least one covid vaccine compared with $77 \%$ in high- and upper-middle-income countries. ${ }^{13}$ Global solidarity and commitment can make this happen; though the vaccine pledge made at the G7 Summit in Cornwall, UK in June 2021, will only reach low-income countries by $2023 .{ }^{14}$ This will prolong the pandemic as low-income countries continue to fight covid-19 outbreaks, allowing the emergence of new variants and spread across the world due to international travel. Low vaccine coverage causes slower economic recovery in emerging markets and developing economies. ${ }^{15}$ Continued research and development of new vaccines effective for new variants are critical measures.

Secondly, thinking of the covid-19 pandemic as a syndemic should help us to focus our efforts on minimising the various determinants which exacerbate the consequences of covid-19 on marginalised and underserved populations. This requires bold leadership across various governments in support of structural reform which addresses inequity through universal social protection, housing, labour, and employment policies. Universal health coverage ensures equitable access by all to healthcare services either in normal and emergency situations. A resilient and equitable recovery requires an equal development path, and comprehensive policy packages on education, healthcare, digital technology and infrastructure.

Thirdly, we must strengthen health systems capacity in terms of preparedness for future epidemics or pandemics. This requires active surveillance of coronavirus and other high impact viral families including influenza, filo- and flavi-viruses in bats and other wildlife worldwide. We must strengthen our diagnostic capacities to detect, sequence and share genomes which strengthen global preparedness and alert. ${ }^{16}$ We must invest in our healthcare workforce capacity, notably epidemiologists and public health officers to respond to outbreaks, as well as frontline clinicians.

Recovery from the covid-19 pandemic requires effective governance for health and solidarity at a global level and leadership, good governance, and 


\section{OPINION}

\section{full involvement of people disproportionately affected by the pandemic.}

Competing interests: none declared

Acknowledgment: This paper is part of the BMJ collection launched at the Prince Mahidol Award Conference 2022 "The World We Want: Actions Towards a Sustainable, Fairer and. Healthier Society"

Provenance and peer review: commissioned, not peer reviewed

1 Agyemang C, Richters A, Jolani S, etal. Ethnic minority status as social determinant for COVID-19 infection, hospitalisation, severity, ICU admission and deaths in the early phase of the pandemic: a meta-analysis. BMJ Glob Health 2021;6:e007433.

doi: 10.1136/bmigh-2021-007433. pmid: 34740916

2 Clift AK, Coupland CAC, Keogh RH, etal. Living risk prediction algorithm (QCOVID) for risk of hospital admission and mortality from coronavirus 19 in adults: national derivation and validation cohort study. BMJ 2020;371:m3731. doi: 10.1136/bmj.m3731. pmid: 33082154

3 Razai MS, Kankam HKN, Majeed A, Esmail A, Williams DR. Mitigating ethnic disparities in covid-19 and beyond. BMJ2021;372:m4921. doi: 10.1136/bmj.m4921. pmid: 33446485

4 Tai DBG, Shah A, Doubeni CA, Sia IG, Wieland ML. The Disproportionate Impact of COVID-19 on Racial and Ethnic Minorities in the United States. Clin Infect Dis 2021;72:703-6. doi: 10.1093/cid/ciaa815. pmid: 32562416

5 Thacker PD. The US politicisation of the pandemic: Raul Grijalva on masks, BAME, and covid-19. BMJ 2020;370:m3430. doi: 10.1136/bmj.m3430. pmid: 32900758

6 Stroebe W, vanDellen MR, Abakoumkin G, etal. Politicization of COVID-19 health-protective behaviors in the United States: Longitudinal and cross-national evidence. PLoS One 2021;16:e0256740. doi: 10.1371/journal.pone.0256740. pmid: 34669724

7 Gover AR, Harper SB, Langton L. Anti-Asian Hate Crime During the COVID-19 Pandemic: Exploring the Reproduction of Inequality. Am J Crim Justice 2020;1-21. doi: 10.1007/s12103-020-09545-1. pmid: 32837171

8 Hahm HC, Ha Y, Scott JC, Wongchai V, Chen JA, Liu CH. Perceived COVID-19-related anti-Asian discrimination predicts post traumatic stress disorder symptoms among Asian and Asian American young adults. Psychiatry Res 2021;303:114084.

doi: 10.1016/j.psychres.2021.114084. pmid: 34242971

9 Islam N, Shkolnikov VM, Acosta RJ, etal. Excess deaths associated with covid-19 pandemic in 2020: age and sex disaggregated time series analysis in 29 high income countries. BMJ 2021;373:n1137. doi: 10.1136/bmj.n1137. pmid: 34011491

10 Kapitsinis N. The underlying factors of excess mortality in 2020: a cross-country analysis of pre-pandemic healthcare conditions and strategies to cope with Covid-19. BMC Health Serv Res 2021;21:1197. doi: 10.1186/s12913-021-07169-7. pmid: 34736434

11 Haldane V, De Foo C, Abdalla SM, etal. Health systems resilience in managing the COVID-19 pandemic: lessons from 28 countries. Nat Med 2021;27:964-80. doi: 10.1038/s41591-021-01381-y. pmid: 34002090

12 Cronin CJ, Evans WN. Excess mortality from COVID and non-COVID causes in minority populations. Proc Natl Acad Sci U S A2021;118:e2101386118. doi: 10.1073/pnas.2101386118. pmid: 34544858

13 The New York Time, Tracking Coronavirus Vaccinations Around the World, Updated Jan. 22, 2022 https://www.nytimes.com/interactive/2021/world/covid-vaccinations-tracker.html

14 Nature. COVID vaccines to reach poorest countries in 2023 - despite recent pledges, news 9 July 2021. doi: 10.1038/d41586-021-01762-w

15 World Bank. Global Economic Prospects, January 2022. World Bank, 2022, doi: 10.1596/978-1-4648-1758-8.

16 Furuse Y. Genomic sequencing effort for SARS-CoV-2 by country during the pandemic. Int I Infect Dis 2021;103:305-7. doi: 10.1016/j.ijid.2020.12.034. pmid: 33333251 